\title{
Social Network Characteristics and Smoking Index Among Smoking Cessation Outpatients in Kunming's Grade-A Tertiary Hospitals in China: A Cross-Sectional Observational Study
}

\section{Rui Zeng}

Second Affiliated Hospital of Kunming Medical University

\section{Li Ai}

Second Affiliated Hospital of Kunming Medical University

\section{Yang Yuan}

Second Affiliated Hospital of Kunming Medical University

\section{Yu Cao}

Second Affiliated Hospital of Kunming Medical University

\section{Ke-Li Chen}

Second Affiliated Hospital of Kunming Medical University

\section{Ran Li}

Second Affiliated Hospital of Kunming Medical University

\section{Xiao-Jie He}

Second Affiliated Hospital of Kunming Medical University

Bing Hai ( $\sim$ 1910645481@qq.com )

Second Affiliated Hospital of Kunming Medical University

\section{Research Article}

Keywords: social network, smoking index, smoking cessation outpatients, Grade-A tertiary hospitals

Posted Date: November 30th, 2021

DOI: https://doi.org/10.21203/rs.3.rs-1010345/v1

License: (1) This work is licensed under a Creative Commons Attribution 4.0 International License. Read Full License 


\section{Abstract}

Introduction

The success rate of smoking cessation outpatients remains modest. Previous studies have highlighted the importance of social networks for quitting smoking, yet there is a substantial lack of studies examining participants in outpatient smoking cessation programs, especially regarding their smoking index.

Aim

We aimed to identify the associations between the social network characteristics of smoking cessation outpatients and their smoking indexes. The association was analyzed with participants in Grade-A Tertiary Hospitals in Kunming, the capital of Yunnan province in China.

\section{Methods}

A multicenter cross-sectional survey was conducted in Kunming in six randomly sampled Grade-A tertiary hospitals. Participants included 351 smoking cessation outpatients who provided data on cigarette smoking and social networks. Multivariate logistic regression was used to examine the association between social network characteristics and smoking index across outpatients. Strong associations were identified using adjusted odds ratios and a $95 \%$ confidence interval.

Results

Large network size $(A O R=1.79,95 \% \mathrm{Cl}=0.99,3.86)$, having children $(A O R=6.35,95 \% \mathrm{Cl}=2.26,19.86)$, and at least one highly influential person in the network $(A O R=2.74,95 \% \mathrm{Cl}=1.13,7.01)$ were all associated with the risk of a high smoking index. However, having drinking friends $(\mathrm{AOR}=0.28,95 \% \mathrm{Cl}=0.14,0.56)$, non-smoking and non-drinking friends in the network $(\mathrm{AOR}=0.44,95 \% \mathrm{Cl}=0.21,0.88)$, and a network member who provided health advice $(\mathrm{AOR}=0.41,95 \% \mathrm{Cl}=0.11,1.35)$ were associated with a lower risk of a high smoking index.The results were sustained even after adjusting for demographic details.

\section{Conclusions}

The study findings suggest the importance of social network characteristics for smoking cessation physicians when formulating a personalized smoking cessation plan for outpatients.

\section{Introduction}

In China, the smoking rate is high. The Chinese report states that there are more than 300 million smokers throughout the nation ${ }^{[1]}$, and the World Health Organization reports that cigarette smoking causes approximately 1 million Chinese deaths annually ${ }^{[2]}$. Smoking damages people's health and is also one of the main risk factors driving the growing prevalence of non-communicable diseases globally ${ }^{[3]}$.

Smoking cessation clinics is the foremost institution that provides smoking cessation services in China. As of 2019, there were 366 hospitals and primary health care centers in 31 provinces with smoking cessation clinics 
in China ${ }^{[4]}$. According to the latest statistics from Yunnan Province, 26 smoking cessation clinics have been established in that region ${ }^{[5]}$. Smoking cessation clinics have been shown to have a positive impact on tobacco control. Bai Hongmei et al. found that smokers' continuous smoking cessation rates over periods of seven days, one month, and three months were $63.4 \%, 29.7 \%$, and $22.8 \%$, respectively [6]. Previous literature reported that the success cessation rate of Chinese smoking cessation clinics is $28.0-30.3 \%$, which is lower than the $57 \%$ reported abroad.

Scholars have found that conventional smoking cessation services and/or medications cannot wholly solve patients' Fagerstrom Test for Nicotine Dependence score, especially those involving social networks ${ }^{[7,8]}$. Therefore, it is necessary for physicians treating patients to help them quit smoking to comprehensively evaluate and understand outpatients' smoking behavior in conjunction with social networks. Social networks is composed of a person's social interaction and a personal relationship network ${ }^{[9]}$. It is not a simple onedimensional plane network but a complex and multidimensional spatial network. Many observational studies have established that social networks are related to healthy behaviors. Social network interventions have been used to reduce weight, improve schizophrenics' health, and treat HIV infection ${ }^{[10,11]}$. Social networks also play an important role in smoking behavior ${ }^{[12][15][16]}$. Pallav Pokhrel et al. found that social network size, perceived social support, and smoking rates were negatively correlated ${ }^{[13]}$. Bonnie Khanh HaBuı et al. confirmed that for non-smokers who do not discuss health issues with network members, the risk of becoming a heavy smoker was 1.097 times higher; for smokers with more intimate relationships, it was 1.531 times higher ${ }^{[14]}$.

In short, there has been research on the association between social networks and smoking behavior (smoking amount, smoking rate, smoking status, willingness to quit, and motivation to quit), but, across the board, there is little or no information about associations with the smoking index. The lack of research is relevant because the smoking index is an important measurement used in smoking cessation clinics ${ }^{[17,18]}$. At present, there are few domestic Chinese studies on social networks and smoking behavior, especially in Yunnan Province. Yunnan Province's tobacco industry was the central economic pillar. Thus, the number of smokers remains high, and smoking cessation clinics are difficult to manage. However, there is a lack of research in this area. Therefore, it is necessary to study the influence of smoking cessation outpatients' social networks on the smoking index in Grade-A Tertiary Hospitals Kunming, the capital city of the province, to provide a basis for formulating personalized smoking cessation intervention strategies.

\section{Methods}

\section{Research Design and Procedures}

This cross-sectional study was conducted in Kunming in 2020. Baseline data of outpatients from six Grade-A tertiary hospitals. They were randomly selected between October 2020 and December 2020. Exclusion criteria included (1) outpatients who went to the smoking cessation clinic by mistake and had not come to quit smoking; (2) outpatients who were unwilling to complete the questionnaire; (3) those with mental illness who could not answer correctly or complete the questionnaire; and (4) not a first-time outpatient. Twelve welltrained research assistants conducted face-to-face interviews with smoking cessation outpatients in six smoking cessation clinics. They were Master's students from Kunming Medical University. They first used 
questionnaires to collect general information, smoking behavior, social networks, and other information. The questionnaire required approximately 30 minutes to complete.

\section{Ethics}

The study was approved by the Medical School Review Board of Kunming Medical University(J-2020-89). The outpatients all signed informed consent, they were given detailed information about completing the form and were informed as to the purpose of the study. All collected data were kept confidential. Confirm that all methods were performed in accordance with relevant guidelines/regulations.

\section{Measures}

\section{Smoking Index}

Smoking behavior was derived from the questionnaire responses and used to assign smoking indexes. The calculated scores the average number of cigarettes smoked per day $\times$ years of tobacco smoking ${ }^{[19]}$. The international medical standard use, the smoking index is divided into four levels: $0=$ non-smoking, $1=$ index score of score $\leq 100,2$ = a score of 101-200, and level 3 applies to a score $>200$. A high smoking index score refers to a smoking index level of 3 (>200).

\section{Social Network}

The questionnaire collected data from the perspective of the personal-centered social network using the four primary aspects of social network structure: social network relationship types, social support methods, social influence, and closeness. First, the outpatients are guided to identify all the people they know well and everyone who knows them well. Then, they identify the people with whom they had contact in the past month through face-to-face communication, phone calls, or any other channel. Finally, from this list, each outpatient identified the five most connected people in their social networks. The detailed characteristics of each network person, including their relationship with the outpatient, whether they smoke, and other demographic characteristics; the type of social support and the degree of influence or closest to the patient, such as whether they support smoking, share cigarettes, and discuss health problems together.

The portion concerning the social network includes the following open-ended questions: (1) Mental support: "If you want to talk about something very personal and private, would you talk to him/her? (Yes/No);" (2) Financial support: "If you want to borrow 1,000 yuan, will he/she lend it to you? (Yes/No);"(3) Health advice: "If you need advice on health issues, will he/she provide advice? (Yes/No);" (4)"What is your relationship with the person?(Spouse, children, other relatives, friends who drink alcohol, friends who smoke, friends (nonsmoking, non-drinking), colleagues, classmates, medical staff, leaders);"(5) "(What is the ) education level of this person?(Illiterate,elementary school, junior high school, high school, university and above);"(6) "How close are you to this person?(Not close at all, a little close, close, very close);"(7) "How much influence does this person have on you?"scored from 1 to 5 points ( 1 = not affect me at all; 5 = can change my decision); (8) "Do you discuss smoking with this person?(No, sometimes, always discuss);" (9) "Does this person share cigarettes with you? (No, yes)"; (10)Does this person support you smoking? (No support, support);" and (11)“Does this person think that smoking is harmful to your health? (Definitely not, maybe not, maybe yes, must be)." 


\section{Data Analysis}

All data analyses were performed using R software (version4.0.3). Descriptive analysis was applied to describe the demographic data, smoking index, and social network characteristics of smoking cessation outpatients, and the network size was defined as the number of members in a person's social network. We recognized that the characteristics of smoking cessation outpatients determined the degree of connection between them and their social networks. The median of discrete variables was used to describe the structural variables in the social network characteristics according to the characteristics of the index; the homogeneity of gender and education level (the number of people with the same characteristics as the surveyed patients/total number of people) were estimated. The score range was $0-1$ where" 1 "means that $50 \%$ or more of the social network members had the same education level and gender(i.e., the gender and education level are similar among st all of the social network members); ${ }^{\prime \prime}$ "means that more than $50 \%$ of social network members and the surveyed person had different educational levels and genders (i.e., the social network did not have the same gender and educational level as the respondent). Regarding the closeness and influence of a social network, if any network member could provide a higher level of intimacy or influence (intimacy level 3 , influence level 4 or 5),the network was defined as having a "high level of closeness or influence."

Regarding social network function variables, if any network member provided emotional support, financial support, health advice, and evaluation support sources, the network was marked as having that specific function. A logistic regression model was used to test the influence of social network characteristics on the smoking index ( $\leq 200$ or $>200$ ). Adjusted demographic data in the multivariate model included gender, ethnicity, marital status, occupation, and economic income. The analysis excluded records with more than $1 \%$ missing values for any variable. Those variables found to have $p$-values less than 0.05 were considered important predictors in the final model.

\section{Results}

\section{Demographic Data of Smoking Cessation Outpatients}

Table 1 presents the demographic distribution of smoking cessation outpatients. In this study, 360 questionnaires were sent out, and 351 valid questionnaires were returned. The recovery rate was $97 \%$. The average smoking index of the sample was $216.77 \pm 240.94$. The majority were married $(51.85 \%)$, Han (70.94\%), and male (97.45\%). Most outpatients were not religious $(66.39 \%)$ and had a high level of education $(67.24 \%)$. The percentage of participants engaged in non-service industries was the largest (81.20\%). The four income levels were nearly equally represented among the participants with the smallest (lowest income) group making up $19.66 \%$ of the sample and the largest (highest income) group accounting for $29.34 \%$. 
Table 1

Demographic distribution data of smoking cessation outpatients by hospital; $n(\%)$.

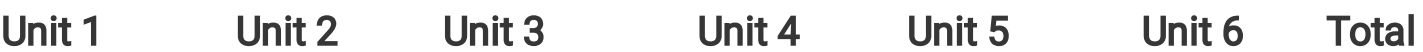

\section{Gender}

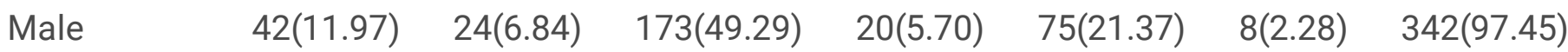

$\begin{array}{llllllll}\text { Female } & 0(0.00) & 0(0.00) & 9(2.55) & 0(0.00) & 0(0.00) & 0(0.00) & 9(2.55)\end{array}$

\section{Religion}

$\begin{array}{llllllll}\text { Buddhism } & 7(1.99) & 2(0.57) & 42(11.97) & 1(0.28) & 8(2.28) & 0(0.00) & 60(17.09) \\ \text { Christianity } & 0(0.00) & 0(0.00) & 6(1.71) & 0(0.00) & 0(0.00) & 0(0.00) & 6(1.71) \\ \text { Catholicism } & 0(0.00) & 0(0.00) & 1(0.28) & 0(0.00) & 0(0.00) & 0(0.00) & 1(0.28) \\ \text { Islam } & 0(0.00) & 0(0.00) & 20(5.71) & 0(0.00) & 2(0.57) & 1(0.28) & 23(6.55) \\ \text { None } & 34(9.69) & 20(5.70) & 93(26.50) & 19(5.41) & 60(17.1) & 7(1.99) & 233(66.39) \\ \text { Others } & 1(0.28) & 2(0.57) & 20(5.71) & 0(0.00) & 5(1.42) & 0(0.00) & 28(7.98)\end{array}$

\section{Education}

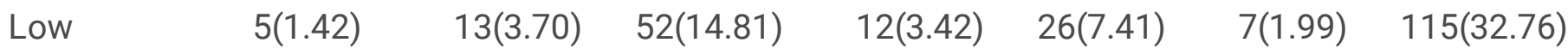

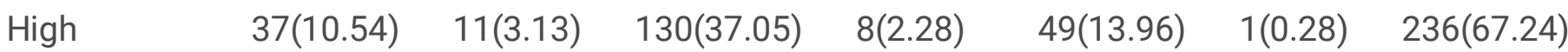

\section{Marital status}

Single $\quad 12(3.24) \quad 14(3.99) \quad 88(25.07) \quad 8(2.28) \quad 36(10.26) \quad 1(0.28) \quad 159(45.30)$

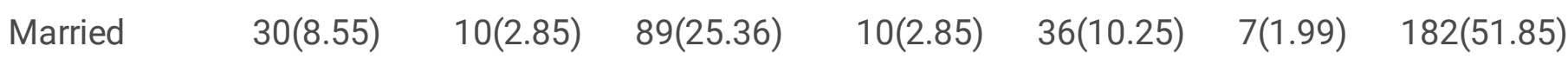

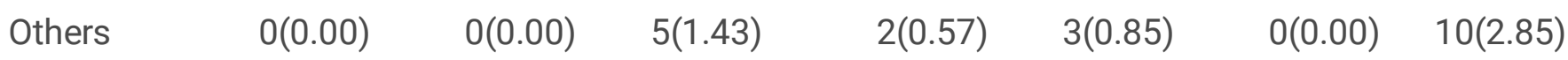

\section{Ethnic group}

$\begin{array}{llllllll}\text { Yi } & 1(0.28) & 2(0.57) & 13(3.71) & 2(0.57) & 6(1.71) & 0(0.00) & 0(0.00) \\ \text { LiSu } & 0(0.00) & 0(0.00) & 7(1.99) & 0(0.00) & 0(0.00) & 0(0.00) & 7(1.99) \\ \text { Hui } & 0(0.00) & 0(0.00) & 16(4.56) & 0(0.00) & 3(0.85) & 0(0.00) & 19(5.41) \\ \text { Han } & 39(11.12) & 19(5.41) & 109(31.06) & 17(4.84) & 58(16.52) & 7(1.99) & 249(70.94) \\ \text { Miao } & 0(0.00) & 0(0.00) & 23(6.27) & 0(0.00) & 1(0.28) & 0(0.00) & 24(6.84) \\ \text { Others } & 2(0.57) & 3(0.86) & 15(4.27) & 1(0.28) & 7(1.99) & 1(0.28) & 28(7.98)\end{array}$

\section{Career}

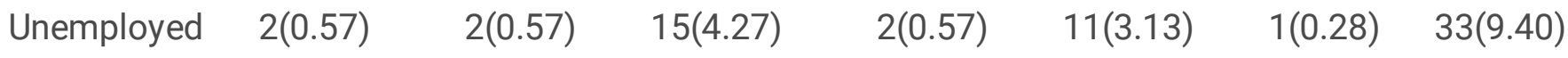

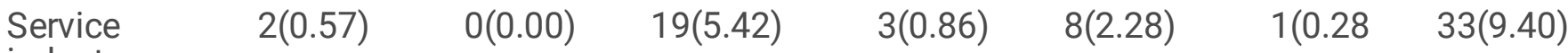




\begin{tabular}{|cccccccc}
\hline & Unit 1 & Unit 2 & Unit 3 & Unit 4 & Unit 5 & Unit 6 & Total \\
\hline $\begin{array}{c}\text { Non-service } \\
\text { industry }\end{array}$ & $38(10.28)$ & $22(6.27)$ & $148(42.17)$ & $15(4.27)$ & $56(15.96)$ & $6(1.71)$ & $285(81.20)$ \\
\hline \multicolumn{2}{|c|}{ Economic income (yuan/month) } & & & & & & \\
\hline$\leq 2000$ & $2(0.57)$ & $5(1.42)$ & $30(8.55)$ & $7(1.99)$ & $24(6.84)$ & $\leq$ & $2(0.57)$ \\
\hline 2000 & $2(0.57)$ & $5(1.42)$ & $30(8.55)$ & $7(1.99)$ & $24(6.84)$ & $\leq$ & $2(0.57)$ \\
\hline $4000-6000$ & $9(2.56)$ & $5(1.42)$ & $56(15.96)$ & $3(0.86)$ & $16(4.56)$ & $9(2.56)$ & $5(1.42)$ \\
\hline$>6000$ & $22(6.27)$ & $5(1.42)$ & $51(14.53)$ & $4(1.14)$ & $21(5.98)$ & $0(0.00)$ & $103(29.34)$ \\
\hline
\end{tabular}

\section{Social Network Characteristics}

Table 2 shows the social network characteristics of the smoking cessation outpatients. Regarding network structure, 351 outpatients reported having a total of 1703 members in their close social network (average 4.85 \pm 0.29 ), and outpatients with the same gender and education level accounted for $83.48 \%$ and $62.39 \%$, respectively. Regarding network relationship type, most were non-smoking and drinking friends (68.09\%), followed by friends who had other relatives (44.44\%), colleagues (39.89\%), and drinking friends $(35.90 \%)$. Regarding the degree of closeness and influence between outpatients and network members, $98.58 \%$ of outpatients had at least one close or very close member, $86.04 \%$ of outpatients had at least one very influential member, $94.02 \%$ of outpatients had members who discussed smoking with them, and $89.17 \%$ of outpatients had members who shared cigarettes and supported their smoking. Regarding social support, the majority believed that at least one of their members would provide financial support (93.45\%), emotional support (97.72\%), health advice (87.18\%), and evaluation support (98.29\%). 
Table 2

Characteristics of the social network of smoking cessation outpatients $(\mathrm{N}=351)$

Social network characteristics

Structure

Size (mean $\pm S D)$

$4.85 \pm 0.29$

Same gender

293(83.48)

Same education level

219(62.39)

\section{Relationship types}

Has an in-network spouse

66(18.80)

Has in-network children

$32(9.12)$

Has otherr in-network relatives

156(44.44)

Has in-network friends who drink alcohol

126(35.90)

Has in-network friends who smoke

$71(20.23)$

Has in-network friends who do not smoke and drink

239(68.09)

Has an in-network colleague

140(39.89)

Has an in-network schoolmate

69(19.66)

Has in-network medical staff

Has an in-network leader

13(3.70)

\section{Closeness and influence}

At least one close or very close person in-network

346(98.58)

At least one very influential person in-network

302(86.04)

Has someone in-network with whom they discuss smoking

$330(94.02)$

Has someone in-network who shares cigarettes and supports smoking

$313(89.17)$

\section{Perceived type of support}

Financial support

$328(93.45)$

Emotional support

343(97.72)

Health advice

306(87.18)

Evaluation support

\section{Relationship Between Social Network and Smoking Index}

As shown in Table 3, social network characteristics significantly associated with smoking indexes were large network size, having children, having drinking friends, and having non-smoking and non-drinking friends in- 
network. Other factors that increased the association were having at least one very influential person in one's social network and getting health advice from network members. In addition, large network size and having children in the network were risk factors for a high smoking index. However, after adjusting for gender, ethnicity, marital status, occupation, and personal income, the association between the large network size and a high smoking index decreased, but the correlation between having children and a high smoking index increased. Beyond that, having at least one very influential person in the network changed the association between the social network and a high smoking index significantly.

Having in-network friends who drink alcohol, friends who do not smoke, friends who do not drink alcohol, and network members who provide health advice were all protective factors against having a high smoking index. Similarly, after adjusting for the above factors, the associations were stronger between higher smoking index scores and having in-network friends who drink alcohol; the index and having friends who neither smoke nor drink were also strongly correlated. However, the association was weaker between smoking index scores and getting health advice from network members (Table 3). 
Table 3

Regression results of social network characteristics related to smoking index (logistic regression predicts smoking index: high and low)

Characteristic of the network

\section{Risk factors}

Network size

Has children in the network

At least one very influential in the network

Protective factors: yes vs. no

Friends who drink

Friends who neither smoke nor drink

Health advice

Non-significant network factors: yes vs. no

Same gender

Same education level

Spouse

Friends who smoke

Colleague

Schoolmate

Medical staff

Leader

Relatives

One close or very close person

Emotional support

Financial support

Evaluation support

Someone who discusses smoking together

Someone who shares cigarettes and supports smoking

\section{Crude OR (95\% Cl) Adjusted OR (95\%}

$\mathrm{Cl})^{\mathrm{a}}$

\begin{tabular}{|c|c|}
\hline $.89(1.07,3.99)^{\star}$ & $1.79(0.99,3.86)^{\star}$ \\
\hline $\begin{array}{l}4.51(1.74 \\
13.02)^{\star \star}\end{array}$ & $6.35(2.26,19.86)^{\star \star}$ \\
\hline $2.17(0.96,5.12)$ & $2.74(1.13,7.01)^{*}$ \\
\hline
\end{tabular}

$2.17(0.96,5.12) \quad 2.74(1.13,7.01)$ * 


\section{Discussion}

This study was the first to evaluate the impact of smoking cessation outpatient social networks on the smoking index in Kunming, the capital and largest city of Yunnan province in China. This article reports the influence of smoking cessation outpatients' social networks on their smoking indexes and provides the most current data on social network structure and functional characteristics. We found multiple associations between social networks and the smoking indexes of smoking cessation outpatients at Kunming's Grade-A Tertiary Hospitals.

\section{Comparisons with Prior Work}

We found that the risk of a high smoking index increased 1.79 times for every increase in the size of the close social network for the smoking cessation outpatients in this study. This finding was consistent with those of Ennett et al., who also reported that social network size was significantly positively correlated with the number of smokers who had smoked in the prior three months ${ }^{[20]}$. First, we speculated that a more extensive social network would be accompanied by a broader range of smoking information and/or more smoking-related resources, which some prior studies have supported ${ }^{[21]}$. In our study, 351 smoking cessation outpatients reported an average network size of 4.85 , of which the proportion of smokers was $89.17 \%$. Second, a larger network potentially provides more social network relationship types; thus, larger networks had a relatively greater impact on healthy behaviors. For example, social network relationships can hinder healthy lifestyles ${ }^{[22]}$. According to a previous study, female smokers who were closely related to other smokers had a 51.52 times higher risk of developing heavy smoking habits, and their odds of quitting smoking were significantly reduced $(\mathrm{OR} 0.74 ; 95 \% \mathrm{Cl}=0.63,0.87)^{[23]}$.

We also found that outpatients with children in the network were at 6.346 times greater risk of having a high smoking index than those without children, which seems to correspond to findings of previous studies that have shown that raising children is one of the main sources of parental pressure, especially since the implementation of China's second-child policy ${ }^{[24,25]}$. For parents, smoking provides a way to manage bad moods and relieve stress. Eriksen testified that parents with more than one child in their social network smoked 10 more cigarettes (or more)per day than parents without children ${ }^{[26]}$. A comparative study of 69 parents of children with developmental disabilities and 137 parents of healthy children showed that the pressure level of the two parents increased, and having children was positively correlated with them being smokers ${ }^{[27]}$.From the above research, we reasonably speculated that, within the network, smoking cessation outpatients who have children would have higher financial and other child-related pressures. Therefore, they would be likely to relieve the pressures of parenting by smoking or increasing the number of cigarettes smoked, leading to a higher smoking index, suggesting that physicians treating patients for smoking cessation should be aware of outpatients with children.

We found that if there was at least one very influential person in someone's social network, the risk of a high smoking index was 2.738 greater than if there was no very influential person. Our research results supported those of Mercken, Dong-Chul, Khalil, Georges, and others ${ }^{[28-30]}$ that highlight the impact of social influence as an integral element of networks. In other words, the premise that social influence encourages network 
members to adopt or adapt to certain behaviors was supported by the research showing that people changed their behaviors and shared activities after spending time together. Many studies have reported that social influence is related to both positive and negative health behaviors, such as smoking, drinking, drug use, and diet/weight-related behaviors ${ }^{[31-34]}$. Our study highlighted the importance of having at least one very influential person in the network to prevent a high smoking index in smoking cessation clinics.

Interestingly, we also found that having drinking friends, non-smoking and non-drinking friends in the network were all associated with a lower probability of a high smoking index. By contrast,other studies have reported that having drinking friends in the network was a risk factor for smoking behavior ${ }^{[35,36]}$. In a study of 630 students with an average age of 16.2 years, Ritchey et al. found that having drinking friends increased the possibility of these students became smokers $(\beta=0.23)$, and the peer pressure to drink alcohol was the main reason for the students becoming smokers $(\beta=0.14){ }^{[37]}$. We observed that the current research object in the relationship between alcohol-drinking friends and smoking behavior in the network is primarily adolescents. Previous studies have also suggested that personal values play a key role in adolescents' smoking behavior. Instead of adults having relatively stable values, they obtained more information about unhealthy behaviors from different sources and were relatively less affected by drinking friends. Regarding whether alcoholdrinking friends provided more health advice that protected outpatients against smoking (reducing the smoking index of smoking cessation outpatients), we did not further stratify research on drinking friends. It was recommended that the population a stratified study conducted with this population would be better for illustrating this problem and should include non-smoking and non-drinking friends in the network sharing to examine good social relationships.

Some studies have shown that good social relationships have a positive impact on the health-related behaviors of respondents ${ }^{[38]}$. Healthy behaviors among network members help people have better and more active health behaviors, thereby reducing both the risk of smoking and the number of cigarettes ${ }^{[39]}$. In our study, having alcohol-drinking friends, non-smoking friends, and non-drinking friends were all related to a lower probability of a high smoking index, indicating that the smoking behaviors of in-network friends can profoundly impact smoking behavior. This result is consistent with the research conclusions of Rostila et al. They deemed that friends' behavior played an important role in the network; in particular, friends with healthy behaviors have a positive impact on the network ${ }^{[40]}$.

Moreover, we found that the ability to get health advice from network members was also associated with a lower probability of a high smoking index, corresponding with the findings of Khanh HaBul and colleagues who reported that being able to discuss health issues with network members helps reduce smoking (OR = $0.555, p<.05)^{[41]}$. Health advice is considered one of the most cost-effective interventions for smoking cessation interventions ${ }^{[42]}$. Ralph et al. found that $25 \%$ of outpatients accepted their doctor's advice, which reduced frequent smokers $\left(36 \%\right.$ ) and frequent cannabis users $(27 \%)^{[43]}$. This finding may help improve the effectiveness of health advice in smoking cessation clinics. In the future, smoking cessation clinics can add health education courses focusing on health advice to benefit smoking cessation clinics.

\section{Limitations and prospects}


There are several limitations to this study. First, this was a cross-sectional survey, so the results can only be used to consider the possibility of causality, and we cannot make any causal inferences. Second, their social networks are undoubtedly larger than the five people they were limited to for nomination in the questionnaire. Third, the measure is also limited to evaluating cigarette use among those in smoking cessation clinics; the use of other tobacco products (such as e-cigarettes and water pipes) is not examined. Future research will benefit from further stratification studies with a longitudinal study that can follow up on the influence of social networks on smoking cessation clinic patients over time. Future studies should also investigate the impact of social networks on the use of other tobacco products (such as e-cigarettes and water pipes) in smoking cessation clinics.

\section{Conclusion}

The results of this study also demonstrate the association between social network characteristics and the smoking indexes of smoking cessation outpatients at Kunming's Grade-A Tertiary Hospitals. Physicians helping patients quit smoking should be aware of the complexity of the influence of social networks. The findings of this study can serve as a rationale for developing individualized smoking cessation programs and interventions that focus on strengthening social networks. For example, including emotional module courses and interpersonal relationship processing skills in health education for smoking cessation outpatients could help them manage the pressures of raising children; it may also help them reduce negative impacts and increase the positive effects of influential people in their networks.

\section{Declarations}

Acknowledgements

This study was a multicenter survey, We thank the center directors and students who participated in the study.

\section{Author Contributions}

Rui Zeng and Li Ai are co-first author, Rui Zeng initiated the study, assisted with data analysis, and wrote the article. Li Ai aspects of the study implementation and contributed to the interpretation and writing of the article. Xiao-Jie He contributed to the study design and analytic plan, performed data analysis, and revised the final draft of the manuscript. Bing Hai supervised all Rui Zeng et al. Yuan Yang, Yu Cao, Ke-Li Chen, Ran $\mathrm{Li}$,and Kai-Jun Ding assisted in data collection. All authors reviewed drafts of the manuscript and approved the version for publication.

\section{Funding}

This study was supported by the in-hospital clinical project of the Second Affiliated Hospital of Kunming Medical University chaired by Professor Hai Bing. The project name is the study on the relationship between the characteristics of social support network and smoking behavior of smoking cessation outpatients in the third class hospital of Kunming(2020ynlc003).

Conflicts of Interest 
None declared.

Consent for publication

Not applicable.

Availability of data and materials

The datasets used and/or analysed during the current study are available

from the corresponding author on reasonable request.

\section{References}

1. The National Health Commission and the WHO Representative in China jointly released the "Report on the Health Risks of Smoking in China 2020." Available at: http://www.chinacdc.cn/jkzt/mxfcrjbhsh/jswj/202107/t20210722_232133.html

Accessed December 5, 2021.

2. Manila, the Philippines, the unaffordable price of China: the health, economic and social losses caused by the tobacco epidemic in China: World Health Organization Western Pacific Regional Office, 2017.

Available at: https://creativecommons.org/licenses/by-nc-sa/3.0/igo

3. Catherine Owens Johnson,Minh Nguyen,Gregory A Roth,Emma Nichols,. Global, regional, and national burden of stroke, 1990-2016: a systematic analysis for the Global Burden of Disease Study 2016. The Lancet Neurology.2019;18:439-458.

4. Lin Haoxiang,Xiao Dan,Liu Zhao,Shi Qiang,Hajek Peter,Wang Chen. National survey of smoking cessation provision in China. Tobacco induced diseases.2019;17:25.

5. Xiangyun Smoking Cessation Clinic is open. Yunnan Provincial Population and Health Promotion and Education Center, Department of Tobacco Control and Psychological Counseling, Accessed December 8, 2021.

6. Bai Hongmei, Cai Haiqing, Li Likang, Jiang Mengying. Study on the smoking cessation status and intervention effects of patients in the pilot hospitals for smoking cessation clinics in Hainan Province in 2015. China Health Education, 2019;35: 138-141+165.

7. Aschbrenner Kelly A,Bobak Carly,Schneider Emily J,Naslund John A,Brunette Mary F,O'Malley A James. Egocentric social networks and smoking among adults with serious mental illness. Translational behavioral medicine.2018;8:531-539.

8. Glorian Sorensen,Karen Emmons,Mary Kay Hunt,Elizabeth Barbeau,Roberta Goldman,Karen Peterson,Karen Kuntz,Anne Stoddard,Lisa Berkman. Model for incorporating social context in health behavior 
interventions: applications for cancer prevention for working-class, multiethnic populations. Preventive Medicine.2003;37:188-197.

9. Mustafa Emirbayer. Manifesto for a Relational Sociology. Mustafa Emirbayer.1997;103:281-317.

10. Van Woudenberg Thabo J.,Bevelander Kirsten E.,Burk William J.,Smit Crystal R.,Buijs Laura,Buijzen Moniek. Testing a Social Network Intervention Using Vlogs to Promote Physical Activity Among Adolescents: A Randomized Controlled Trial. Frontiers in Psychology.2020:542.

11. Giuseppe Carrà,Claudia Battaglia,Mark Hinton,Luke Sheridan Rains,Cristina Crocamo,Sonia Johnson. Social network confidants, duration of untreated psychosis and cannabis use in people with first episode psychosis: An exploratory study. Early Intervention in Psychiatry.2018;12:942-946.

12. Julie Neisler,Lorraine R. Reitzel,Lorra Garey,Darla E. Kendzor,Emily T. Hébert,Maya

Vijayaraghavan,Michael S. Businelle. Corrigendum to "The moderating effect of perceived 29 social support on the relation between heaviness of smoking and quit attempts among adult homeless smokers" [Drug and Alcohol Depend. Drug and Alcohol Dependence.2019;199:128-132.

13. Pokhrel Pallav,Fagan Pebbles,Cassel Kevin,Trinidad Dennis R,Kaholokula Joseph Keawe'aimoku,Herzog Thaddeus A. Social Network Characteristics, Social Support, and Cigarette Smoking among Asian/Pacific Islander Young Adults. American journal of community psychology.2016;57:353-65.

14. Bonnie Khánh Hà Buì. Personal Social Networks in Later-Life Alcohol and Cigarette Use.Substance Use \& Misuse.2020;55:1905-1911.

15. E Hwa Yun,Yoon Hwa Kang,Min Kyung Lim,Jin-Kyoung Oh,Jung Min Son. The role of social support and social networks in smoking behavior among middle and older aged people in rural areas of South Korea:A cross-sectional study. BioMed Central.2010;10:78.

16. Roberts Megan E, Bernstein Michael H, Colby Suzanne M. The effects of eliciting implicit versus explicit social support among youths susceptible for late-onset smoking.2016;62:60-4.

17. Laurie Zawertailo,Sabrina Voci,Peter Selby. The Cigarette Dependence Scale and Heaviness of Smoking Index as predictors of smoking cessation after 10 weeks of nicotine replacement therapy and at 6-month follow-up.Addictive Behaviors.2018;78:223-227.

18. Abhaya Indrayan Dr,Rajeev Kumar Mr,Shridhar Dwivedi Dr.A SIMPI E INDEX OF SMOKING.cobra preprint series.2008.

19. Federal Government. Salud, SEDENA, SEMAR. Clinical Practice Guidelines.Prevention,diagnosis and treatment of smoking and second-hand smoke at the first level of care (Guía de Práctica Clínica. Prevención, diagnóstico y tratamiento del consumo de tabaco. 2009;13:94.

20. Susan T. Ennett,Robert Faris,John Hipp,Vangie A. Foshee,Karl E. Bauman,Andrea Hussong,Li Cai. Peer Smoking, Other Peer Attributes, and Adolescent Cigarette Smoking: A Social Network Analysis. Prevention Science.2008;9:88-98. 
21. Marcel Alejandro de Dios,Cassandra A. Stanton,Celeste M. Caviness,Raymond Niaura,Michael Stein. The Social Support and Social Network Characteristics of Smokers in Methadone Maintenance Treatment. The American Journal of Drug and Alcohol Abuse.2013;39:50-56.

22. John C. Barefoot,Morten GrønbÆEk,Gorm Jensen,Peter Schnohr,Eva Prescott. Social Network Diversity and Risks of Ischemic Heart Disease and Total Mortality: Findings from the Copenhagen City Heart Study.American Journal of Epidemiology.2005;161:960-7.

23. Ari Väänänen,Anne Kouvonen,Mika Kivimäki,Jaana Pentti,Jussi Vahtera. Social Support, Network Heterogeneity, and Smoking Behavior in Women: The 10-Town Study. American Journal of Health Promotion.2008;22:246-55.

24. Cheng Hongmei. Talking about the source of parental pressure in second-child families. Modern Communication, 2019: 65+64.

25. Li Xiaowei, Xie Juan, Song Yating. The characteristics of parent-parent co-parenting and its relationship with mothers' parenting pressure and child problem behaviors. China Special Education, 2016: 71-78.

26. Willy Eriksen. Social support and the smoking behaviour of parents with preschool children.Scandinavian Journal of Public Health.1997;25:93-9.

27. Chakraborty Bhaswati,Rao Arathi,Shenoy Ramya,Davda Latha,Suprabha B S.Stress-mediated quality of life outcomes in parents of disabled children: A case-control study.Journal of the Indian Society of Pedodontics and Preventive Dentistry.2019;37:550-557.

28. Liesbeth Mercken,Math Candel,Paul Willems,Hein De Vries. Disentangling social selection and social influence effects on adolescent smoking: the importance of reciprocity in friendships.Addiction.2007;102:1483-92.

29. Seo Dong-Chul,Huang Yan. Systematic review of social network analysis in adolescent cigarette smoking behavior. The Journal of school health.2012;82:21-7.

30. Khalil Georges E.,Jones Eric C.,Fujimoto Kayo. Examining proximity exposure in a social network as a mechanism driving peer influence of adolescent smoking. Addictive 31 Behaviors.2021;117:106853.

31. Vítor P. Lopes,Carl Gabbard,Luis P. Rodrigues. Physical Activity in Adolescents: Examining Influence of the Best Friend Dyad. Journal ofAdolescent Health.2013;52:752-6.

32. I.-Chen Lee,Te-Tien Ting,Duan-Rung Chen,Fang-Yi Tseng,Wei J. Chen,Chuan-Yu Chen.Peers and social network on alcohol drinking through early adolescence in Taiwan. Drug and Alcohol Dependence.2015;153:508.

33. Fujimoto Kayo,Valente Thomas W. Decomposing the components of friendship and friends' influence on adolescent drinking and smoking. The Journal of adolescent health:official publication of the Society forAdolescent Medicine.2012;51:136-43. 
34. Shen L, Assanangkornchai S, Liu W, Cai L, Li F, Tang S, et al. Influence of social network on drug use among clients of methadone maintenance treatment centers in Kunming, China. PLoS ONE.2018;13: e0200105.

35. Leung RK, Toumbourou JW, Hemphill SA. The effect of peer influence and selection processes on adolescent alcohol use: a systematic review of longitudinal studies. Health psychology review. 2014; 8:42657.

36. Seo D-C, Huang Y. Systematic Review of Social Network Analysis in Adolescent Cigarette Smoking Behavior*. Journal of School Health. 2012; 82:21-7.

37. Phillip N. Ritchey,Gerald S. Reid,Lora A. Hasse. The relative influence of smoking on drinking and drinking on smoking among high school students in a rural tobacco-growing county. Journal ofAdolescent Health.2001;29:386-94.

38. Lisa F. Berkman,Thomas Glass,Ian Brissette,Teresa E. Seeman. From social integration to health: Durkheim in the new millennium This paper is adapted from Berkman, L.F., \& Glass,T. Social integration, social networks, social support and health. In L. F. Berkman \& I. Kawachi, Social Epidemiology . New York: Oxford University Press; and Brissette, I., Cohen S., Seeman, T. Measuring social integration and social networks. In S. Cohen, L. Underwood \& B. Gottlieb, Social Support Measurements and Intervention . New York: Oxford University Press. Social Science \& Medicine.2000;51:843-857.

39. Mikael Rostila. Birds of a feather flock together - and fall ill? Migrant homophily and health in Sweden. Sociology of Health \& IIIness.2010;32:382-99.

40. Rostila,Mikael,Almquist,Ylva B,Östberg, Viveca,Edling, Christofer,Rydgren, Jens. Social Network Characteristics and Daily Smoking among Young Adults in Sweden. International Journal of Environmental Research and Public Health.2013;10:6517-33.

41. Bonnie Khánh Hà Buì. Personal Social Networks in Later-Life Alcohol and Cigarette Use.Substance Use \& Misuse.2020;55:1905-1911.

42. Cobos-Campos Raquel,Apiñaniz Fernández de Larrinoa Antxon,Sáez de Lafuente Moriñigo Arantza,Parraza Diez Naiara,Aizpuru Barandiaran Felipe. Effectiveness of Text Messaging as an Adjuvant to Health Advice in Smoking Cessation Programs in Primary Care. A Randomized Clinical Trial. Nicotine \& Tobacco Research.2017;19:901-907.

43. Hingson Ralph W,Zha Wenxing,lannotti Ronald J,Simons-Morton Bruce. Physician advice to adolescents about drinking and other health behaviors. Pediatrics.2013;131:249-57. 